Bundesgesundheitsbl 2018 $61: 1422-1428$ https://doi.org/10.1007/s00103-018-2823-y Online publiziert: 4. Oktober 2018

(c) Der/die Autor(en) 2018

CrossMark

Elke Pieper - Nadja Mallock • Frank Henkler-Stephani · Andreas Luch

Abteilung Chemikalien- und Produktsicherheit, Bundesinstitut für Risikobewertung (BfR), Berlin, Deutschland

\title{
Tabakerhitzer als neues Produkt der Tabakindustrie: Gesundheitliche Risiken
}

Die gesundheitsbezogenen Risiken des Tabakrauchens entstehen durch Karzinogene und andere gesundheitlich bedenkliche Emissionen. Ansätze zur Schadstoffreduktion für konventionelle Zigaretten sind daher sinnvoll und werden auch von der Weltgesundheitsorganisation (WHO) weiter verfolgt [5]. Die Einführung von unabhängigen Untersuchungseinrichtungen, Messmethoden, Informationspflichten sowie die Schadstoffregulierung gründen sich letztlich auf diesem Konzept. Allerdings bestehen auch Grenzen. Beim konventionellen Rauchen gibt es kaum Möglichkeiten, die Verbrennungstemperatur $\mathrm{zu}$ senken und die technischen Möglichkeiten zur weiteren Minimierung gesundheitsschädlicher Emissionen sind möglicherweise ausgeschöpft. Tabakerhitzer könnten daher als neue Technologie betrachtet werden, die diese Grenze verschieben kann. In den neuen Geräten wird ein Tabakstick beispielsweise mittels eines elektrischen Heizdorns oder Heizblatts auf etwa $350^{\circ} \mathrm{C}$ erhitzt. Diese Temperatur liegt deutlich unter den $900^{\circ} \mathrm{C}$, die in der Glutkuppe einer Tabakzigarette erreicht werden. Dies führt zu verminderten Schadstoffemissionen, wobei die Nikotinfreisetzung im Bereich einer konventionellen Tabakzigarette liegt. Tabakerhitzer könnten einen wichtigen Beitrag leisten, um die Schadstoffemissionen von Rauchtabak wirksam zu begrenzen, auch wenn die Auswirkungen auf gesundheitliche Risiken nicht abschließend bewertet werden können. Statt Schadstoffreduktionen von 50-60\%, die bei konventionellen Zigaretten erreichbar waren, besteht nun die technische Möglichkeit, wichtige kanzerogene Substanzen in den Emissionen um mehr als $80-99 \%$ zu reduzieren. Bisherige Minimierungsstrategien der WHO bezogen sich jedoch ausschließlich auf traditionelle Zigaretten und nicht auf technische Neuentwicklungen, die häufig als Modified- oder Reduced-Exposure/Risk-Geräte bezeichnet werden. Aus toxikologischer Sicht erscheint es dagegen sinnvoll, Minimierungsansätze mit neuen Technologien zu verbinden. Andererseits bestehen Bedenken, dass Tabakerhitzer den irreführenden Eindruck eines nahezu unbedenklichen Tabakkonsums wecken könnten und neben den verbleibenden, substanziellen Schadstoffemissionen auch ein hohes Suchtpotenzial bergen.

In Deutschland kam 2016 das erste Modell in den Handel, das in der Fachliteratur als Tabakerhitzer THS 2.2 (Tobacco Heating System 2.2) bezeichnet wird. Der Hersteller führte dazu umfangreiche Studien zur Verminderung der schädlichen und potenziell schädlichen Stoffe (HPHC: „harmful and potentially harmful constituents") in den Emissionen durch. Weitere Untersuchungen legten nahe, dass durch die geringere Exposition auch das Gesundheitsrisiko gesenkt wird, wenn man komplett von der konventionellen Tabakzigarette auf den Tabakerhitzer umsteigt [6-9]. Allerdings sind für eine Risikobewertung dieses neuen Produktes unabhängige standardisierte Daten notwendig, um Exposition und gesundheitliche Risiken abzuschätzen. Laut WHO fehlen unabhängige Studien, die belegen, dass eine verringerte Exposition gegenüber gesundheitsschäd- 


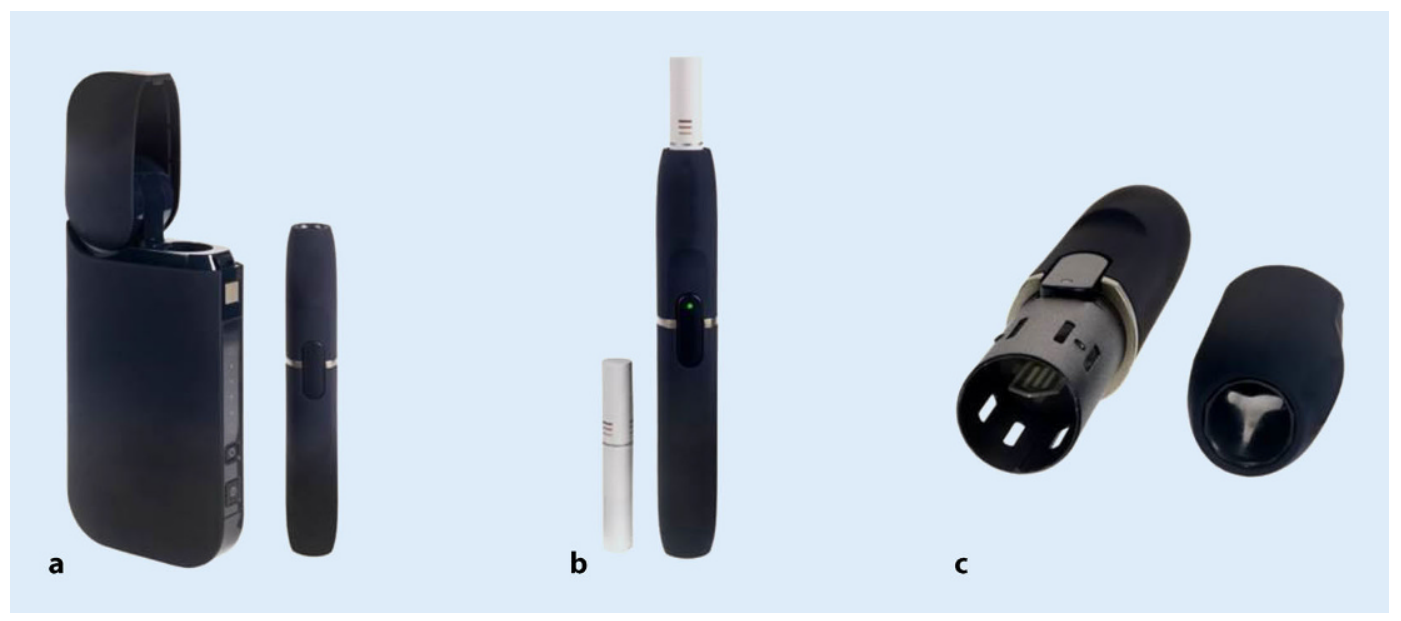

Abb. $1<$ a Ladegerät (links) und Holder (rechts), b Tabakstick (links) und Holder mit Tabakstick (rechts), cHolder mit sichtbarem Heizblatt (links) und Kappe des Holders (rechts)

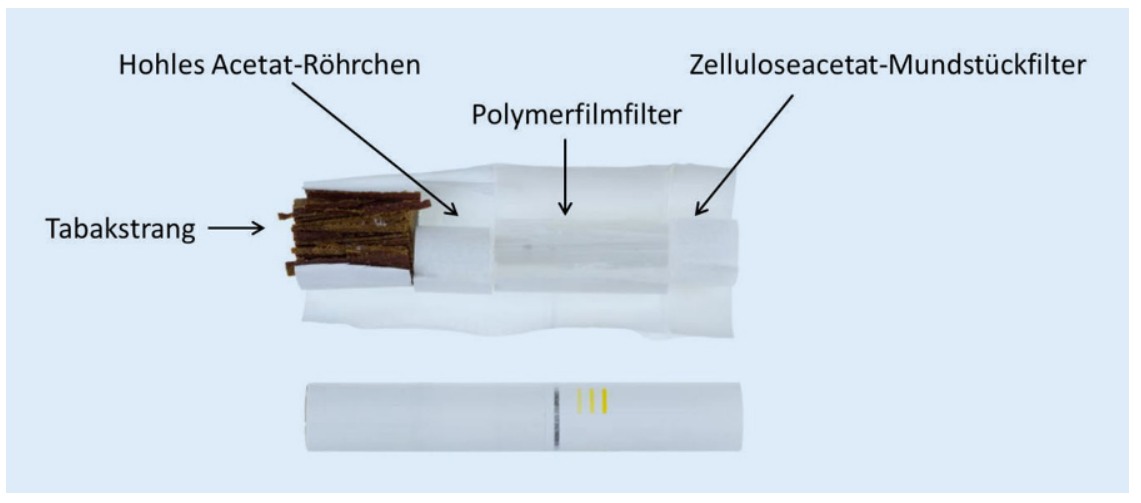

Abb. 2 A Oben Querschnittansicht eines Tabaksticks, unten intakter Tabakstick in gleicher Orientierung

lichen Stoffen durch die Verwendung von Tabakerhitzern auch zu einem geringeren Risiko führen [10]. Das Bundesinstitut für Risikobewertung (BfR) und andere Institutionen haben in ersten Studien die Reduktion ausgewählter schädlicher bzw. potenziell schädlicher Stoffe in den Emissionen bestätigt. Der Nikotingehalt in den Emissionen des Tabakerhitzers ist vergleichbar mit dem konventioneller Zigaretten. Daher ist von einem vergleichbaren Sucht- und Abhängigkeitspotenzial auszugehen. Aus diesem Grund ist jedoch auch kein Kompensationsverhalten, d.h. ein Ausgleich der geringeren Nikotinaufnahme durch häufigeres oder intensiveres Rauchen zu erwarten. Bisher gibt es nur wenige Untersuchungen und Bewertungen zu den Auswirkungen auf gesundheitliche Risiken. In den USA läuft zurzeit ein umfangreiches Einstufungsverfahren, welches über die Anerkennung dieses Modells als Modified Risk Tobacco Product (MRTP) entschei- det. Dieser Prozess schließt auch eine umfassende Bewertung der Schadstoffreduktion ein [11]. Ziel des vorliegenden Beitrags ist es, aktuelle Studien über den in Deutschland erhältlichen Tabakerhitzer sowie eine möglicherweise verringerte Gesundheitsgefährdung zu diskutieren.

\section{Was sind Tabakerhitzer und wie funktionieren sie?}

Tabakerhitzer sind batteriebetriebene Systeme, die durch elektrisches Erhitzen von Tabak nikotinhaltige Emissionen erzeugen. Dafür werden Tabaksticks in einem entsprechenden Heizgerät platziert und auf etwa $250-350^{\circ} \mathrm{C}$ erwärmt. Dabei entstehen nikotinhaltige Emissionen, die über ein Mundstück mit Filtersegment inhaliert werden.

Der in Deutschland erhältliche Tabakerhitzer besteht aus 3 Komponenten mit unterschiedlichen Funktionen
(• Abb. 1). Dazu zählt der Tabakstick mit verarbeitetem Tabak, ein stiftartiges Heizgerät (Holder), in das der Tabakstick gesteckt wird, welcher dann mittels eines elektrisch kontrollierten Heizstabes erhitzt wird, sowie ein Ladegerät (der Charger), das das Heizgerät nach Gebrauch wieder auflädt. Der Tabakerhitzer beendet den Heizprozess automatisch nach 6 min oder 14 Zügen, sodass Pyrolyseprodukte und Schadstofffreisetzung sowohl zeitlich als auch durch eine maximale Zugzahl pro Stick limitiert sind. Der Tabakstick enthält einen komprimierten Tabakstrang sowie mehrere Filterelemente (• Abb. 2). Der Tabakstrang besteht aus einer getrockneten Tabaksuspension, die zu einer papierdünnen braunen Tabakfolie ${ }^{1}$ aufgerollt wurde. Diese setzt sich aus ca. $70 \%$ Tabak sowie Feuchthaltemitteln, Bindemitteln und Aromastoffen zusammen. Als Feuchthaltemittel werden Wasser und Glycerin verwendet, um ein Austrocknen zu verhindern und um die Aerosolbildung beim Erhitzen zu unterstützen [12]. Die Filterelemente bestehen aus 2 unabhängigen Systemen: einem Polymerfilmfilter, der das Aerosol abkühlt, gefolgt von einem weichen Zelluloseacetatmundstückfilter, welcher die sensorischen Aspekte einer konventionellen Zigarette imitiert [12].

\footnotetext{
1 Tabakfolie (rekonstituierte Tabakfolie) - Tabakstaub, der durch Fasern und Bindemittel zu einer Folie verarbeitet wird, die anstelle von verarbeitetem Rohtabakin Zigaretten und Zigarren entsprechend § 18 Tabakerzeugnisgesetz eingesetzt werden darf.
} 
Bundesgesundheitsbl 2018 · 61:1422-1428 https://doi.org/10.1007/s00103-018-2823-y

(c) Der/die Autor(en) 2018

E. Pieper · N. Mallock · F. Henkler-Stephani · A. Luch

Tabakerhitzer als neues Produkt der Tabakindustrie: Gesundheitliche Risiken

\section{Zusammenfassung}

Die verstärkten Maßnahmen in der Tabakkontrolle der letzten Jahre führten dazu, dass die Tabakindustrie mit der Entwicklung neuer Tabakprodukte, wie Tabakerhitzer, reagiert, deren Konsum weniger gesundheitsschädlich als der von konventionellen Zigaretten sein soll. Es liegen umfangreiche Studien seitens der Hersteller vor, die aufzeigen, dass die Emissionen des Tabakerhitzers deutlich geringere Werte an gesundheitsschädlichen Substanzen im Vergleich zu konventionellen Zigaretten aufweisen. Im Weiteren veröffentlichten Hersteller Studien, die verminderte toxische Wirkungen im Vergleich zur herkömmlichen Zigarette belegen.

Ziel des vorliegenden Berichtes ist es, aktuelle Studien des Herstellers und unabhängiger
Institutionen zu erörtern sowie mögliche verringerte Gesundheitsgefährdungen zu diskutieren.

Das Bundesinstitut für Risikobewertung (BfR) hat eine eigene Studie zu ausgewählten Analyten in den Emissionen eines Tabakerhitzers durchgeführt, welche die niedrigeren Gehalte an gesundheitsschädlichen Stoffen in den Emissionen bestätigt. Dies wurde auch in anderen unabhängigen Studien gezeigt. Der Nikotingehalt in den Emissionen liegt im gleichen Bereich wie die Nikotinemissionen von konventionellen Zigaretten, wodurch von einem vergleichbaren Sucht- und Abhängigkeitspotenzial auszugehen ist. Der Hersteller hat mutagene Wirkungen der Emissionen berichtet, die allerdings im
Vergleich zu konventionellen Zigaretten deutlich schwächer ausgeprägt sind. Dennoch bleibt die Nutzung von Tabakerhitzern mit gesundheitlichen Risiken verbunden. Es ist noch unklar, inwieweit die reduzierten Gehalte an gesundheitsschädlichen Stoffen zu einem geringeren gesundheitlichen Risiko führen. Dazu sind zum einen mehr unabhängige Studien, aber auch Langzeitstudien notwendig.

Schlüsselwörter

Zigaretten - Emissionen · Schadstoffreduktion . Tabakrauch · Passivrauchen

\section{"Heat not burn" tobacco devices as new tobacco industry products: health risks}

\section{Abstract}

Increased tobacco control measures in recent years have directed the tobacco industry to develop alternative tobacco products, such as "heat not burn" ( $\mathrm{HnB})$ tobacco devices that are implied to be less hazardous than conventional cigarettes. There are extensive studies from manufacturers available, which show that the emissions of $\mathrm{HnB}$ tobacco devices have significantly lower levels of harmful substances compared to conventional cigarettes. In addition, manufacturers have published studies to investigate whether switching from a conventional tobacco cigarette to the $\mathrm{HnB}$ product reduces possible health risks.

The purpose of this report is to review current studies by manufacturers and independent institutions as well as to discuss possible reduced health hazards.

The German Federal Institute for Risk Assessment (BfR) has carried out its own studies of selected analytes in the emissions of one $\mathrm{HnB}$ product confirming the lower levels of harmful substances in the emissions. The results are consistent with data from other independent studies. The nicotine content in the emissions is in the same range as the nicotine emissions of conventional cigarettes, which suggests a comparable addictiveness and dependence potential. Manufacturers have reported mutagenic effects of emissions by $\mathrm{HnB}$ tobacco devices that, however, are considerably weaker compared to conventional cigarettes. Nevertheless, the use of the $\mathrm{HnB}$ tobacco product remains associated with health risks.

Switching from conventional cigarettes to tobacco heaters can significantly reduce the consumer's exposure to harmful substances. However, this article also illustrates that it is still unclear to what extent the reduced levels lead to lowered health risks. Therefore more independent studies, but also long-term studies, are needed.

Keywords Cigarettes - Emissions - Harm reduction . Tobacco smoke $\cdot$ Secondhand smoking

\section{Was ist Zigarettenrauch und wie entstehen gesundheitlich bedenkliche Stoffe?}

Konventionelle Tabakzigaretten sind sehr gut untersuchte und standardisierte Produkte. Zigarettenrauch ist ein Aerosol, das aus ca. 4800 Verbindungen besteht, wovon 89 als gesundheitlich gefährlich eingestuft wurden [13]. Bei jedem Zug werden in der Glutzone Temperaturen von ungefähr $900^{\circ} \mathrm{C}$ erreicht, wobei der Tabak verbrennt. Unter Sauerstoffmangel kommt es im angrenzenden
Tabakstrang zu endothermen Reaktionen, die das organische und anorganische Material thermisch zersetzen. Die gasförmigen Reaktionsprodukte gelangen in die Destillationszone, wo die Temperatur entsprechend der Entfernung zur Glutzone auf $600-200^{\circ} \mathrm{C}$ abkühlt. Hier entstehen die meisten gesundheitlich bedenklichen Stoffe. Durch Verdampfen der flüchtigen Anteile und Destillation entsteht das Aerosol. Dabei gehen niedrig siedende Stoffe direkt in den Rauch über. Hinter der Destillationszone befindet sich die Kondensationszone, wo ein Teil des Rauches wieder kondensiert. Der inhalierte Hauptstromrauch besteht daher aus einer partikulären sowie einer Gasphase [14].

Unter Passivrauchen versteht man die Aufnahme von Tabakrauch aus der Raumluft oder der Umwelt, der $\mathrm{zu} 80-85 \%$ aus Nebenstromrauch und zu $15-20 \%$ aus wieder ausgeatmetem Hauptstromrauch besteht. Der Nebenstromrauch entsteht durch Destillation beim Glimmen bei etwa $500^{\circ} \mathrm{C}$ in den Zugpausen. Da mehr Material destilliert als verbrannt wird, unterscheidet sich 


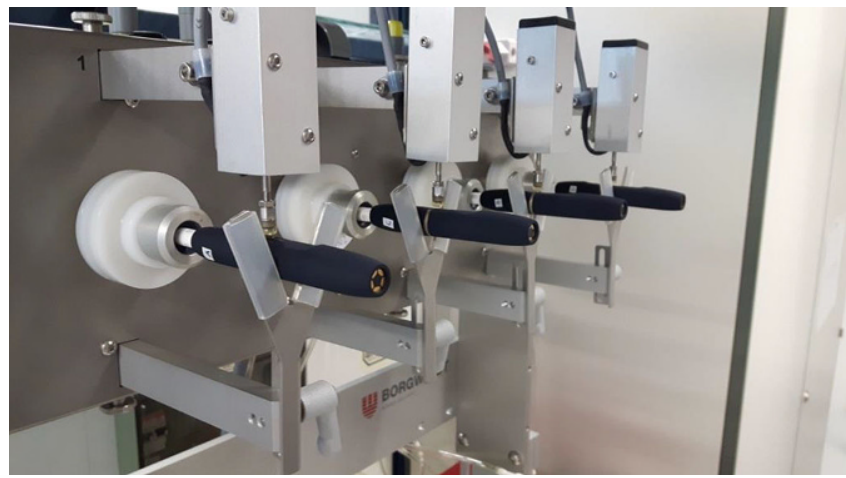

Abb. $3 \triangleleft$ E-Zigarettenabrauchmaschine mit Tabakerhitzern. Die Emissionen von 4 Tabaksticks können parallel in einem $A b$ rauchexperiment generiert werden

der Neben- vom Hauptstromrauch in seiner Zusammensetzung.

\section{Vergleich der Emissionen des Tabakerhitzers mit konventionellen Zigaretten}

Das BfR hat kürzlich in Zusammenarbeit mit dem Chemischen und Veterinäruntersuchungsamt (CVUA) Sigmaringen eine erste Studie zu ausgewählten Analyten in den Emissionen von Tabakerhitzern abgeschlossen [15]. Dabei wurde ein standardisiertes Abrauchverfahren (Health Canada Intense Smoking Regimen, HCI; [16]) an einer E-Zigarettenabrauchmaschine verwendet (siehe - Abb. 3), um den Hauptstromrauch zu generieren. Obwohl Abrauchmaschinen nicht zu $100 \%$ das menschliche Rauchverhalten widerspiegeln, stellen sie doch eine gute Näherung dar. Die erzeugten Emissionen werden mithilfe von geeigneten analytischen Methoden untersucht, wodurch ein Vergleich von verschiedenen Applikationen und Produkten ermöglicht wird. In der Studie des BfR wurden Partikelphase, Nikotin, Wasser sowie Aldehyde und flüchtige organische Substanzen, zu denen wichtige Hauptkanzerogene gehören [17], analysiert. Die verwendeten Methoden kommen in Routinelaboratorien zum Einsatz, was für eine spätere Qualitätssicherung und Überwachung von Bedeutung ist. Die Daten anderer unabhängiger Studien sowie des Herstellers wurden bestätigt. Die Emissionen im Hauptstromrauch des Tabakerhitzers sind in - Tab. 1 im Vergleich zum Hauptstromrauch konventioneller Zigaretten dargestellt. Für konventionelle Zigaretten sind die höchsten und die niedrigsten Werte aus der Studie von Counts et al. angegeben [18]. Zwischen einzelnen Zigarettenmarken gibt es erhebliche Schwankungen, sodass die formale Berechnung der Schadstoffreduktion auch stark von der verwendeten Referenzzigarette abhängt. Der Nikotinwert des Tabakerhitzers ist vergleichbar mit den Werten von konventionellen Zigaretten. Der Wert für die Gesamtpartikelmasse liegt teilweise höher. Allerdings hat die Gesamtpartikelmasse der Tabakerhitzer eine andere Zusammensetzung, da der Anteil von Wasser und Feuchthaltemitteln deutlich höher ist. Vergleicht man den Gehalt der Carbonylverbindungen Formaldehyd, Acetaldehyd, Acrolein und Crotonaldehyd, erhält man für die Emissionen des Tabakerhitzers eine deutliche Reduktion von $80-96 \%$ gegenüber konventionellen Tabakzigaretten. Die Gehalte an flüchtigen und semiflüchtigen Verbindungen in den Emissionen sind im Tabakerhitzer sogar um 97-99\% niedriger im Vergleich zu konventionellen Zigaretten. Die Daten der BfR-Studie sind in guter Übereinstimmung mit Daten aus weiteren unabhängigen Studien, die sich ebenfalls auf ausgesuchte Analyten im Hauptstromrauch des THS 2.2 fokussierten [19-21].

\section{Weiterführende Studien zum Tabakerhitzer}

Es wurde eine Reihe von In-vitro-, Invivo- und klinischen Studien [22] veröffentlicht, die zumeist im Rahmen des Einstufungsverfahrens in den USA durchgeführt wurden. Bei der Durchführung dieser Studien hatte sich der Hersteller an validierten Prüfverfahren orientiert. Im Weiteren führte der Hersteller Stu- dien zum Einfluss der Tabakerhitzer auf die Innenraumluftqualität durch [23].

\section{In-vitro- und In-vivo-Toxizität}

Vom Hersteller wurden 6 In-vitro-Toxizitätsstudien durchgeführt. Dabei wurden unter anderem genotoxische und mutagene Eigenschaften der Emissionen von Tabakerhitzern mit den Emissionen der Referenzzigarette 3R4F verglichen [22]. $\mathrm{Zu}$ beachten ist hierbei, dass Referenzzigaretten für analytische Zwecke entwickelt wurden und hohe Schadstoffgehalte in den Emissionen aufweisen, die oft oberhalb der Werte von handelsüblichen Zigaretten liegen. Studien mit Referenzzigaretten können daher für neue Tabakprodukte stärker verminderte Emissionen oder Effekte ergeben als ein Vergleich mit Marktzigaretten.

$\mathrm{Zu}$ der In-vitro-Standardtestbatterie für Genotoxizität gehören der AmesTest sowie der Maus-Lymphoma-Test. Der Ames-Test ist ein bakterieller reverser Mutagenitätstest, bei dem $\mathrm{Mu}$ tationen rückgängig gemacht werden, wodurch essenzielle Aminosäuren wieder synthetisiert werden können. In den untersuchten Bakterienstämmen zeigten die Emissionen des Tabakerhitzers kein mutagenes Potenzial an. Im Maus-Lymphoma-Test, bei dem die Mutationshäufigkeit in einem speziellen Säugetiergewebe gemessen wird, wurden vergleichsweise schwache mutagene Wirkungen nachgewiesen. Dabei stieg die Mutationshäufigkeit allerdings erst bei deutlich höherer Exposition im Vergleich zu konventionellen Tabakprodukten an. Die Effekte der Zelltoxizität und pathologische Veränderungen an menschlichem Gewebe waren weniger stark ausgeprägt und traten im Vergleich zum Tabakrauch konventioneller Zigaretten erst bei deutlich höherer Konzentration auf. Trotz der verminderten Freisetzung gesundheitsschädlicher Substanzen bleibt die Nutzung der Tabakerhitzer mit einer Inhalation krebserzeugender Substanzen und von Pyrolyseprodukten verbunden. Bisher ist nicht klar, wie sich die verminderte Schadstoffexposition auf ein potenziell gesenktes Gesundheitsrisiko übertragen lässt. 
Tab. 1 Gehalte an ausgesuchten Analyten im Hauptstromrauch eines Tabakerhitzers und konventioneller Zigaretten. Es sind jeweils die höchsten und niedrigsten Werte angegeben, die von Mallock et al. [15] in 2 verschiedenen Sorten Tabaksticks sowie von Counts et al. [18] in konventionellen Zigaretten gefunden wurden. In Spalte 5 ist die Reduktion der Analyten im Hauptstromrauch des Tabakerhitzers im Vergleich zu konventionellen Zigaretten in \% dargestellt

Tabaksticks
(Mallock et al. 2018;
[15])

Konventionelle Reduktion

Zigaretten

(Counts et al. 2005; [18])

\begin{tabular}{|c|c|c|c|c|}
\hline Parameter & Einheit & Min.-Max. & Min.-Max. & $\%$ \\
\hline Züge & Zug/Tabakstrang & 12 & $5,5-13,6$ & - \\
\hline TPM & mg/Tabakstrang & $51,2-52,6$ & $27,5-60,9$ & - \\
\hline Nikotin & mg/Tabakstrang & 1,1 & $1,07-2,70$ & - \\
\hline Wasser & mg/Tabakstrang & $28,0-31,7$ & $9,8-21,4$ & - \\
\hline NFDPM & mg/Tabakstrang & $19,8-21,6$ & $16,3-37,6$ & - \\
\hline Acetaldehyd & $\mu \mathrm{g} /$ Tabakstrang & $179,4-183,5$ & $930-1540$ & $80,5-88,2$ \\
\hline Acrolein & $\mu \mathrm{g} /$ Tabakstrang & $8,9-9,9$ & $89,2-154,1$ & $89,5-93,9$ \\
\hline Formaldehyde & $\mu \mathrm{g} /$ Tabakstrang & $4,7-5,3$ & $29,3-130,3$ & $82,9-96,2$ \\
\hline Crotonaldehyd & $\mu \mathrm{g} /$ Tabakstrang & $<3,0$ & $32,7-70,8$ & - \\
\hline 1,3-Butadiene & $\mu \mathrm{g} /$ Tabakstrang & $0,20-0,2$ & $77,0-116,7$ & $99,7-99,8$ \\
\hline Benzol & $\mu \mathrm{g} / T a b a k s t r a n g$ & $0,5-0,6$ & $49,7-98,3$ & $98,8-99,4$ \\
\hline Isopren & $\mu \mathrm{g} /$ Tabakstrang & $1,8-2,1$ & 509-1160 & $99,6-99,8$ \\
\hline Styrol & $\mu \mathrm{g} /$ Tabakstrang & 0,5 & $15,4-33,3$ & $96,9-98,6$ \\
\hline Toluol & $\mu \mathrm{g} /$ Tabakstrang & $2,0-2,2$ & $86,2-176,2$ & $97,6-98,8$ \\
\hline \multicolumn{5}{|c|}{$\begin{array}{l}\text { Tabakstrang, } d \text {. h. für Tabakerhitzer: ein Tabakstick; für konventionelle Zigarette: eine Ziga- } \\
\text { rette } \\
\text { Alle Werte wurden unter Verwendung der Health-Canada-Intense(HCI)-Abrauchbedingungen ge- } \\
\text { neriert } \\
\text { TPM Gesamtpartikelmasse („total particulate matter“), NFDPM nikotinfreies Trockenkondensat } \\
\text { („nicotine-free dried particulate matter") }\end{array}$} \\
\hline
\end{tabular}

Im Rahmen des Einstufungsverfahrens wurden In-vivo-Studien in den USA durchgeführt, die ebenfalls eine verminderte Schadstoffexposition bestätigten. Der Einsatz von Tierversuchen zur Entwicklung von Tabakerzeugnissen sollte jedoch hinterfragt werden. In Deutschland besteht ein entsprechendes Verbot.

\section{Klinische Studien}

Der Hersteller führte 4 klinische Studien durch, um zu untersuchen, ob sich eine geringere Exposition positiv auf entsprechende Biomarker und gegebenenfalls auf die Entstehung tabakassoziierter Krankheiten auswirkt [6-9]. Zwei der Studien, die in Polen und Japan durchgeführt wurden, waren stationär durchgeführte 5-tägige Studien $[6,7]$. Dabei wurden 160 Raucher randomisiert in 3 Gruppen eingeteilt: 1) Raucher konventioneller Zigaretten,
2) Raucher, die komplett auf Tabakerhitzer umsteigen, und 3) Raucher, die aufhören $\mathrm{zu}$ rauchen (Abstinenzler). Dabei wurde bereits wenige Tage nach dem Umstieg auf den Tabakerhitzer eine deutliche Reduktion für 16 mit dem Rauchen assoziierte Biomarker im Blut bzw. im Urin gemessen. Die Messungen der Biomarker für 1,3-Butadien (Monohydroxybutenylmercaptursäure, MHBMA), Acrolein (3-Hydroxypropylmercaptursäure, 3-HPMA), Benzol (S-Phenylmercaptursäure, S-PMA) und Acrylnitril (Cyanoethylmercaptursäure, CEMA) ergaben für die in Polen durchgeführte Studie eine Reduktion von entsprechend $92 \%, 59 \%, 94 \%$ und $87 \%$. Im Vergleich zu den verminderten Gehalten in den Emissionen waren die Auswirkungen auf die entsprechenden Biomarker weniger deutlich.

Die beiden anderen Untersuchungen waren 3-monatige Studien und wurden in Japan und den USA durchgeführt $[8,9]$.

\section{Infobox 1 Fakten zum}

Tabakerhitzer

- Da der durch Tabakerhitzer erzeugte Dampf Nikotin enthält, besteht das Risiko einer Nikotinabhängigkeit.

- Die Exposition gegenüber mutagenen und weiteren gesundheitsschädlichen Stoffen ist geringer als bei konventionellen Zigaretten. Eine verringerte Exposition gegenüber gesundheitsschädlichen Stoffen bedeutet allerdings nicht, dass die gesundheitlichen Risiken in gleichem Maße herabgesetzt sind. Selbst eine geringe Exposition erhöht die Risiken für Krebserkrankungen, Schlaganfall und andere Herz-Kreislauf-Erkrankungen im Vergleich zum Nichtraucher. Wie stark das Gesundheitsrisiko für tabakassoziierte Erkrankungen verändert wird, kann daher nicht bewertet werden. Es fehlen Langzeitstudien.

- Die Innenraumluftqualität wird durch die Emissionen von Tabakerhitzern deutlich weniger belastet als durch konventionelle Zigaretten. Trotzdem können für Nichtraucher gesundheitliche Risiken durch Passivrauchen nicht ausgeschlossen werden.

Diese begannen ebenfalls mit einer 5-tägigen stationären Phase mit anschließenden 90 Tagen ambulanter Betreuung. Allerdings fehlen bei den ambulanten Studien quantitative Angaben zur Compliance der Studienteilnehmer. In der in Japan durchgeführten Studie wird die Compliance als „besonders hoch“ beschrieben. Für die in den USA durchgeführte Studie wird eine "gute" Compliance für die Tabakerhitzergruppe berichtet, aber eine „schlechte" für die Abstinenzgruppe, wodurch sich die Variabilität erhöht und dadurch die Interpretation der Ergebnisse unmöglich gemacht wird. Zusätzlich haben frühere Mitarbeiter des Herstellers Unregelmäßigkeiten in der Durchführung der klinischen Studien aufgezeigt [23]. Dies unterstreicht die Notwendigkeit einer unabhängigen Bewertung der Rohdaten.

\section{Innenraumluftqualität und Passivrauchen}

Passivrauchen ist ein wichtiger Risikofaktor für eine Vielzahl von tabakassoziierten Krankheiten [24]. Daher ist es wichtig, den Einfluss von Tabakerhitzern auf 
Nichtraucher zu untersuchen. Die Hersteller untersuchten den Einfluss von Tabakerhitzern auf die Luftqualität im Vergleich zu herkömmlichen Zigaretten. Es wurde kein messbarer Passivrauch im Innenraum gefunden, nur die Werte für Nikotin und Acetaldehyd waren leicht erhöht $[25,26]$. In einer neuen Untersuchung, in der Nichtraucher dem Passivrauch von Tabakerhitzern ausgesetzt waren, berichtet allerdings fast die Hälfte von mindestens einem akuten Symptom durch die Exposition [27]. In einer weiteren unabhängigen Studie wurde gezeigt, dass Tabakerhitzer Kleinstpartikel von einem Mikrometer oder kleiner sowie Nanopartikel emittieren [28]. Tabakerhitzer können in geringem Umfang einen $\mathrm{Ne}$ benstromrauch zwischen einzelnen Zügen bilden [29]. Ruprecht et al. untersuchten den Passivrauch eines Tabakerhitzers und fanden bestimmte n-Alkane, organische Säuren und Anhydrozuckerverbindungen wie Levoglucosan mit bis $\mathrm{zu}$ umgerechnet $2-6 \mathrm{mg} / \mathrm{h}$ während eines normalen Abrauchvorgangs. Im Vergleich zum Passivrauch einer konventionellen Zigarette waren beim Tabakerhitzer feste Partikel mit einem Durchmesser von $1-1000 \mathrm{~nm}$ um $59-62 \%$ reduziert. Im Weiteren wurden Formaldehyd, Acetaldehyd und Acrolein im Passivrauch gefunden, aber in einem deutlich niedrigeren Konzentrationsbereich als bei konventionellen Zigaretten [30].

\section{Fazit}

Aufgrund der vergleichsweise niedrigen Temperaturen, der Filtersysteme und einer neuartigen physikalischen Gestaltung erreichen Tabakerhitzer eine erhebliche Reduktion von gesundheitsschädlichen Emissionen (80-99\%) im Vergleich zu herkömmlichen Tabakzigaretten. Gleichzeitig ermöglichen diese neuen Geräte eine effektive Nikotinaufnahme, die der parallelen oder nur gelegentlichen Nutzung von Tabakzigaretten entgegenwirken kann. Das Suchtpotenzial bleibt bestehen. Tabakerhitzer bergen jedoch nach wie vor gesundheitliche Risiken (s. Infobox 1). Trotz reduzierter Schadstoffgehalte wurden für die Emissionen beispielsweise mutagene Wirkungen dokumentiert, die im Vergleich zum Zigarettenrauch jedoch deutlich schwächer ausgeprägt waren.

Tabakerhitzer unterscheiden sich grundsätzlich von Lightzigaretten („low-yield cigarettes") und ermöglichen tatsächlich eine geringere Schadstoffexposition, die jedoch in langfristig angelegten Untersuchungen weiter charakterisiert werden sollte. Aufgrund einer erheblich reduzierten Schadstofffreisetzung sind verminderte gesundheitliche Risiken zu erwarten, falls auf den Konsum anderer Tabakerzeugnisse ansonsten verzichtet wird. Eine Abschätzung und Bewertung der verbleibenden Risiken für tabakassoziierte Erkrankungen ist derzeit noch nicht möglich und erfordert die Entwicklung entsprechender Modelle. Dafür gibt es mehrere Ansätze: Fowles and Dybing (2003) haben Bestandteile des Hauptstromrauches von konventionellen Tabakzigaretten entsprechend ihrer Konzentration und dem Tumorpotenzfaktor priorisiert [17]. Tumorpotenzfaktoren sind ein Modellierungsansatz, um kanzerogene Eigenschaften von Stoffen zu quantifizieren und zu vergleichen. Bei der Ableitung dieser Faktoren spielen unter anderem kanzerogene Wirkmechanismen sowie Dosis-Wirkungs-Beziehungen aus Tierversuchen und Humandaten eine wichtige Rolle. Auf dieser Grundlage hatte Stephens (2017) die Tumorpotenz der bekannten Emissionen aller Schadstoffe eines Tabakerhitzers zusammenfassend berechnet, die im Vergleich zu Tabakzigaretten auf etwa $10 \%$ reduziert war [31]. Diese Schätzung stimmt mit epidemiologischen Studien überein, die belegen, dass bei einem entsprechend reduzierten Zigarettenkonsum ( $<2$ pro Tag) zwar das Lungenkrebsrisiko sinkt [32], im Vergleich zu Nichtrauchern aber deutlich erhöht bleibt. Für kardiovaskuläre Erkrankungen vermindert sich das Risiko allerdings nicht im gleichen Maße. Eine kürzlich durchgeführte Metaanalyse ergab, dass das Risiko für kardiovaskuläre Erkrankungen beim Konsum von einer Zigarette pro Tag im Vergleich zu 20 Zigaretten pro Tag immer noch bei $40-50 \%$ liegt [33]. Es ist allerdings noch unklar, ob Daten zum reduzierten Zigarettenkonsum auch zur Modellierung von Gesundheitsrisiken durch die verminderte Schadstofffreisetzung von Tabakerhitzern verwendet werden können, da sich beispielsweise die Expositionszeiten erheblich unterscheiden. Für die Entwicklung robuster Bewertungsmodelle sind weitere Untersuchungen und Studien erforderlich. Es ist zu erwarten, dass Tabakerhitzer anderer Hersteller [34-37] in naher Zukunft auf dem deutschen Markt erhältlich sind. Eine Überprüfung der toxikologisch relevanten Rauchinhaltsstoffe durch unabhängige Überwachungslabore wäre somit sinnvoll, um eine Beurteilung der angegebenen reduzierten Schadstoffemissionen für den Konsumenten zu gewährleisten.

\section{Korrespondenzadresse}

\section{Dr. Elke Pieper}

Abteilung Chemikalien- und Produktsicherheit, Bundesinstitut für Risikobewertung (BfR)

Max-Dohrn-Str. 8-10, 10589 Berlin,

Deutschland

elke.pieper@bfr.bund.de

\section{Einhaltung ethischer Richtlinien}

Interessenkonflikt. E. Pieper, N. Mallock, F. HenklerStephani und A. Luch geben an, dass kein Interessenkonflikt besteht.

Dieser Beitrag beinhaltet keine von den Autoren durchgeführten Studien an Menschen oder Tieren.

Open Access. Dieser Artikel wird unter der Creative Commons Namensnennung 4.0 International Lizenz (http://creativecommons.org/licenses/by/4.0/deed. de) veröffentlicht, welche die Nutzung, Vervielfältigung, Bearbeitung, Verbreitung und Wiedergabe in jeglichem Medium und Format erlaubt, sofern Sie den/die ursprünglichen Autor(en) und die Quelle ordnungsgemäß nennen, einen Linkzur Creative Commons Lizenz beifügen und angeben, ob Änderungen vorgenommen wurden.

\section{Literatur}

1. Thun MJ, Heath CW Jr. (1997) Changes in mortality from smoking in two American Cancer Society prospective studies since 1959. Prev Med 26:422-426

2. Djordjevic MV, Hoffmann D, Hoffmann I (1997) Nicotine regulates smoking patterns. Prev Med 26:435-440

3. Hammond D, Fong GT, Cummings KM, Hyland A (2005) Smoking topography, brand switching, and 
nicotine delivery: results from an in vivo study. Cancer Epidemiol Biomarkers Prev 14:1370-1375

4. Song MA, Benowitz NL, Berman M et al (2017) Cigarette filter ventilation and its relationship to increasing rates of lung adenocarcinoma. J Natl Cancer Inst 109:djx75

5. Burns DM, Dybing E, Gray N et al (2008) Mandated lowering of toxicants in cigarette smoke: a description of the World Health Organization TobReg proposal. Tob Control 17:132-141

6. Haziza C, De La Bourdonnaye GD, Skiada D et al (2017) Biomarker of exposure level data set in smokers switching from conventional cigarettes to Tobacco Heating System 2.2, continuing smoking or abstaining from smoking for 5 days. Data Brief 10:283-293

7. Haziza C, De La Bourdonnaye G, Skiada D et al (2016) Evaluation of the Tobacco Heating System 2.2. Part 8: 5-Day randomized reduced exposure clinical study in Poland. Regul Toxicol Pharmacol 2:139-S150

8. Ludicke F, Picavet P, Baker G et al (2018) Effects of Switching to the Menthol Tobacco Heating System 2.2, Smoking Abstinence, or Continued Cigarette Smoking on Clinically Relevant Risk Markers. Nicotine Tob Res 20:173-182 (A Randomized, Controlled, Open-Label, Multicenter Study in Sequential Confinement and Ambulatory Settings (Part 2))

9. Ludicke F, Picavet P, Baker G et al (2018) Effects of switching to the tobacco heating system 2.2 menthol, smoking abstinence, or continued cigarette smoking on biomarkers of exposure: a randomized, controlled, open-label, multicenter study in sequential confinement and ambulatory settings (part 1). Nicotine Tob Res 20:161-172

10. World Health Organization (2018) Heated tobacco products (HTPs) information sheet. http://www. who.int/tobacco/publications/prod_regulation/ heated-tobacco-products/en/. Zugegriffen: 24. Juni 2018

11. Food, Admistration D (2018) FDA briefing document:january24-25,2018TPSAC meeting. https:// www.fda.gov/downloads/AdvisoryCommittees/ CommitteesMeetingMaterials/TobaccoProducts ScientificAdvisoryCommittee/UCM593109.pdf. Zugegriffen: 24. Juli 2018

12. Smith MR, Clark B, Ludicke F et al (2016) Evaluation of the tobacco heating system 2.2. Part 1: description of the system and the scientific assessment program. Regul Toxicol Pharmacol 81(Suppl 2):S17-S26

13. Talhout R, Schulz T, FlorekE, Van Benthem J, Wester P, Opperhuizen A (2011) Hazardous compounds in tobacco smoke. Int J Environ Res Public Health 8:613-628

14. Baker RR, Bishop LJ (2004) The pyrolysis of tobacco ingredients. J Anal Appl Pyrolysis 71:223-331

15. Mallock N, Boss L, Burk R et al (2018) Levels of selected analytes in the emissions of "heat not burn" tobacco products that are relevant to assess human health risks. Arch Toxicol 92:2145-2149

16. Health Canada (2000) Tobacco reporting regulation, SSOR/2000-273

17. Fowles J, Dybing E (2003) Application of toxicological risk assessment principles to the chemical constituents of cigarette smoke. Tob Control 12:424-430

18. Counts ME, Morton MJ, Laffoon SW, Cox RH, Lipowicz PJ (2005) Smoke composition and predicting relationships for international commercial cigarettes smoked with three machine-smoking conditions. Regul Toxicol Pharmacol 41:185-227
19. Auer R, Concha-Lozano N, Jacot-Sadowski I, Cornuz J, Berthet A (2017) Heat-not-burn tobacco cigarettes: smoke by any other name. JAMA Intern Med 177:1050-1052

20. Bekki K, Inaba Y, Uchiyama S, Kunugita N (2017) Comparison of chemicals in mainstream smoke in heat-not-burn tobacco and combustion cigarettes. JUOEH 39:201-207

21. Li X, Luo Y, Jiang $X$ et al (2018) Chemical analysis and simulated pyrolysis of tobacco heating system 2.2 compared to conventional cigarettes. Nicotine TobRes. https://doi.org/10.1093/ntr/nty005

22. Schaller JP, Keller D, Poget L et al (2016) Evaluation of the Tobacco Heating System 2.2. Part 2: Chemical composition, genotoxicity, cytotoxicity, and physical properties of the aerosol. Regul Toxicol Pharmacol 81(Suppl 2):S27-S47

23. Reuters Investigates (2018) Part 3 - Scientists describe problems in Philip Morris e-cigarette experiments. https://www.reuters.com/investigates/ special-report/tobacco-iqos-science/. Zugegriffen: 24. Juni 2018

24. Barnoya J, Navas-Acien A (2013) Protecting the world from secondhand tobacco smoke exposure: where do we stand and where do we go from here? Nicotine Tob Res 15:789-804

25. Mitova MI, Campelos PB, Goujon-Ginglinger CG et al (2016) Comparison of the impact of the Tobacco Heating System 2.2 and a cigarette on indoor air quality. Regul Toxicol Pharmacol 80:91-101

26. Forster M, Mcaughey J, Prasad K, Mavropoulou E, Proctor C (2017) Assessment of tobacco heating product THP1.0. Part 4: characterisation of indoor air quality and odour. Regul Toxicol Pharmacol. https://doi.org/10.1016/j.yrtph.2017.09.017

27. Tabuchi T, Gallus S, Shinozaki T, Nakaya T, Kunugita N, Colwell B (2017) Heat-not-burn tobacco product use in Japan: its prevalence, predictors and perceived symptoms from exposure to secondhand heat-not-burn tobacco aerosol. Tob Control. https://doi.org/10.1136/tobaccocontrol2017-053947

28. Protano C, Manigrasso M, Avino P, Vitali M (2017) Second-hand smoke generated by combustion and electronic smoking devices used in real scenarios: Ultrafine particle pollution and agerelated dose assessment. Environ Int 107:190-195

29. O'connell G, Wilkinson P, Burseg KMM, Stotesbury SJ, Pritchard JD (2015) Heated tobacco products create side-stream emissions: implications for regulation. Int J Environ Anal Chem 2:163

30. Ruprecht AA, De Marco C, Saffari A et al (2017) Environmental pollution and emission factors of electronic cigarettes, heat-not-burn tobacco products, and conventional cigarettes. Aerosol Sci Technol 51:674-684

31. Stephens WE (2017) Comparing the cancer potencies of emissions from vapourised nicotine products including e-cigarettes with those of tobacco smoke. Tob Control. https://doi.org/10. 1136/tobaccocontrol-2017-053808

32. Cancer Prevention Study II (CPSII) (2016) Amercian cancer society

33. Hackshaw A, Morris JK, Boniface S, Tang JL, Milenkovic D (2018) Low cigarette consumption and risk of coronary heart disease and stroke: metaanalysis of 141 cohort studies in 55 study reports. BMJ 360:j5855

34. Proctor C (2018) Assessment of tobacco heating product THP1.0. Part 1: series introduction. Regul Toxicol Pharmacol 93:1-3

35. Eaton D, Jakaj B, Forster M et al (2017) Assessment of tobacco heating product THP1.0. Part 2: product design, operation and thermophysical characterisation. Regul Toxicol Pharmacol. https:// doi.org/10.1016/j.yrtph.2017.09.009

36. Miura N, Yuki D, Minami N, Kakehi A, Futamura $Y$ (2015) A study to investigate changes in the levels of biomarkers of exposure to selected cigarette smoke constituents in Japanese adult male smokers who switched to a non-combustion inhaler type of tobacco product. Regul Toxicol Pharmacol 71:498-506

37. Ishikawa S, Matsumura K, Kitamura N, Ishimori K, Takanami Y, Ito S (2018) Application of a direct aerosol exposure system for the assessment of biological effects of cigarette smoke and novel tobacco product vapor on human bronchial epithelial cultures. Regul Toxicol Pharmacol 96:85-93 